Original Paper http://ajol.info/index.php/ijbcs http://indexmedicus.afro.who.int

\title{
Evaluation nutritionnelle de la morelle africaine au Cameroun
}

\author{
Mara Naomie NGO BOGMIS, Funamo Amos NGWA et Gabriel Ambroise MANGA*
}

Institut de Recherche Agricole pour le Développement (IRAD), BP 13 Njombé, Cameroun.

*Corresponding author; E-mail: ambroisemangag@gmail.com

\section{REMERCIEMENTS}

Ce travail a bénéficié de l'appui financier du Contrat de Désendettement Développement (C2D) à travers le Programme d'Appui à la Recherche (PAR) du projet Horticulture.

\section{RESUME}

Une évaluation nutritionnelle a été effectuée sur les échantillons de morelle africaine collectés dans 25 sites de production du Cameroun. L'objectif était de déterminer l'impact du site sur la qualité nutritive des légumes. Les paramètres examinés étaient : la matière sèche, les cendres, les protéines brutes et les minéraux. Les résultats ont montré une différence significative $(\mathrm{p}<0,05)$ entre les sites. Le taux de matière sèche était compris entre 5 et $32,50 \%$; le taux de cendres moyen variait de 15,50 à 32,29\%; les protéines brutes étaient élevées notamment à l'Ouest et comprises entre $25,63 \%$ et $72,64 \%$. Les minéraux présentaient aussi des valeurs élevées en phosphore $(601$ à $9256 \mathrm{mg} / 100 \mathrm{~g})$, calcium $(430$ à $3485 \mathrm{mg} / 100 \mathrm{~g})$, fer $(1,22$ à 223,21 $\mathrm{mg} / 100 \mathrm{~g})$ et cuivre $(8,93$ à $34,82 \mathrm{mg} / \mathrm{kg})$. Le cuivre dans la plupart des sites présentait des valeurs supérieures à la norme admise par la FAO et pourrait se révéler dangereux pour les consommateurs. Des analyses des sols et des systèmes de cultures pratiqués dans les différents sites pourraient permettre de déterminer les sources potentielles de contamination.

(C) 2018 International Formulae Group. All rights reserved.

Mots clés : Morelle africaine, sites de production, qualité nutritive, Cameroun.

\section{Nutritional assessment of African nightshade in Cameroon}

\begin{abstract}
A nutritional assessment was carried out on African nightshade samples collected from 25 production sites in Cameroon. The objective was to determine the impact of the site on the nutritional quality of vegetables. The parameters examined were: dry matter content, ash, crude protein and minerals. The results showed a significant difference $(\mathrm{p}<0.05)$ between the sites. The dry matter content was between 5 and $32.50 \%$; the average ash rate ranged from 15.50 to $32.29 \%$; crude protein was high, particularly in the West, ranging from $25.63 \%$ to $72.64 \%$. Minerals also portrayed high levels for phosphorus (601 to $9256 \mathrm{mg} / 100 \mathrm{~g}$ ), calcium (430 to $3485 \mathrm{mg} / 100 \mathrm{~g}$ ), iron (1.22 to $223.21 \mathrm{mg} / 100 \mathrm{~g}$ ) and copper (8.93 to $34.82 \mathrm{mg} / \mathrm{kg}$ ). In most of
\end{abstract}


the sites, copper levels were higher than the standard accepted by FAO and could be dangerous for consumers. Analysis of soil and cropping systems at different sites could help identifying potential sources of contamination.

(C) 2018 International Formulae Group. All rights reserved.

Keywords: African nightshade, production sites, nutritional quality, Cameroon.

\section{INTRODUCTION}

Les légumes sont importants dans l'alimentation des populations, car ils contribuent à la sécurité alimentaire par leur composition et constituent une source importante d'emplois et de revenus (AdorglohHessou, 2006). La morelle africaine (Solanum scabrum) est un des légumes-feuilles les plus cultivés en zone urbaine, péri-urbaine et rurale du Cameroun (Batchep, 2009). Elle occupe une place importante grâce à ses qualités nutritionnelles qui contribuent au bien-être de l'organisme humain (Tchiegang et al., 2004 ; Iheanacho et al., 2009).

Les parties comestibles ont une forte teneur en eau qui leur confère un caractère très périssable et une durée de conservation très courte due aux actions des agents biologiques et physico-chimiques de dégradation. La morelle est l'un des légumes traditionnels pour lequel plusieurs auteurs ont rapporté l'importance dans la réduction des problèmes de malnutrition en apportant les quantités requises en protéines, minéraux et vitamines au corps humain (Diouf et al., 2017). Selon l'expérience d'Asian Vegetable Research Development Center (AVRDC), les systèmes de production, le choix des semences et les techniques culturales sont déterminants pour la culture de ces légumes (Tchientche et al., 2013). Cependant, au-delà de leurs contributions positives sur le plan de la sécurité alimentaire et nutritionnelle d'une part et comme source de revenus et d'emplois d'autre part, les systèmes de productions maraîchères présentent des risques sanitaires et environnementaux très élevés (Wognin et al., 2013). En effet, l'usage excessif des engrais et des produits phytosanitaires par les producteurs engendre de nombreux problèmes dont les plus importants sont : la contamination des récoltes par les résidus de pesticides ; la pollution de la nappe phréatique et la périssabilité des productions qui entraîne de nombreuses pertes post-récolte (pourritures). Il importe donc de déterminer les caractéristiques des légumes-feuilles, pour apprécier la qualité de la morelle africaine utilisée par les consommateurs. La présente étude a été menée afin de déterminer la valeur nutritionnelle de la morelle récoltée dans différents sites de production du Cameroun ainsi que l'impact du site sur sa qualité nutritive.

\section{MATERIEL ET METHODES}

\section{Description des zones de collecte}

L'étude a été menée de juillet 2014 à juin 2017 dans 25 sites localisés dans cinq régions (Sud-Ouest, Littoral, Centre, Nord et Ouest) de mise en œuvre du projet IRAD/C2D-PAR Horticulture, représentant quatre des cinq zones agroécologiques du Cameroun dont les caractéristiques biophysiques sont décrites par Ngom et al. (2014).

\section{Matériel végétal}

$\mathrm{La}$ morelle africaine (Solanum scabrum) a été collectée sur des parcelles de production des légumes-feuilles à partir des semences acquises dans les marchés par les producteurs.

\section{Echantillonnage}

Pour chaque site, les tiges fraîches ont été prélevées au hasard sur les pieds de légumes sur différentes parcelles de morelle 
africaine appartenant à un groupe d'acteurs préalablement identifiés. L'échantillon était constitué de feuilles récoltées dans les champs en préfloraison. Une partie de chaque échantillon a été nettoyée à sec puis les feuilles et bourgeons terminaux ont été utilisés pour la détermination de la matière sèche. L'autre partie de l'échantillon rincée à l'eau du robinet et séchée à l'étuve à $70{ }^{\circ} \mathrm{C}$ a été ensuite emballée et acheminée au Laboratoire d'Analyse des Sols, Plantes, Engrais et Eaux (LASPEE) de l'IRAD de Nkolbisson.

L'analyse chimique des échantillons a porté sur la teneur totale en cendres, les protéines brutes et les minéraux $(\mathrm{Ca}, \mathrm{Fe}, \mathrm{P}$, $\mathrm{Cu})$. Les différents paramètres de la morelle africaine notamment le taux de matière sèche, la teneur en cendres étaient déterminés selon les méthodes AOAC (1990). La matière sèche a été obtenue par séchage à l'étuve à $105{ }^{\circ} \mathrm{C}$ jusqu'à l'obtention d'un poids sec constant, puis l'application de l'équation suivante : MS $(\%)=$ (poids sec/poids frais) $\times 100$. La teneur en cendres totales ou matière minérale a été déterminée par pesée directe des matières sèches (échantillons) avant et après calcination au four à moufle à $450{ }^{\circ} \mathrm{C}$ pendant $4 \mathrm{~h}$ jusqu'à apparition des cendres blanches.

Le pourcentage des cendres totales a été obtenu suivant l'équation: \% Cendre = $\left(\left(\mathrm{m}_{2}-\mathrm{m}_{0}\right) /\left(\mathrm{m}_{1}-\mathrm{m}_{0}\right)\right) \times 100$.

Les protéines brutes ont été réalisées par la méthode Kjeldahl, la teneur en protéines a été calculée en multipliant la teneur en azote total par 6,25.

La détermination de la composition minérale a été effectuée en dissolvant $1 \mathrm{~g}$ des cendres dans $5 \mathrm{ml} \mathrm{du}$ mélange $(50 \%$ eau + $50 \%$ acide Chlorhydrique concentrée) et en lisant les absorbances au spectrophotomètre UV Visible (JENWAY) à émission optique couplé à un plasma induit (ICP-OES). Les concentrations calculées sont en $\mathrm{g} / \mathrm{kg}$ de matière sèche pour le Phosphore et le
Calcium, en $\mathrm{mg} / \mathrm{kg}$ de matière sèche pour le Cuivre et le Fer.

\section{Analyse statistique}

Le traitement des données a été réalisé avec Excel 2013 et l'analyse statistique avec SPSS version 21. Au seuil de signification $\mathrm{p}<$ $5 \%, 1$ 'ANOVA a permis d'examiner la valeur alimentaire de la morelle provenant des différents sites de production. Lorsque des différences significatives étaient décelées sur les paramètres mesurés (protéines, minéraux) le test de Duncan a été réalisé afin de séparer les moyennes des échantillons de morelle africaine des différents sites.

\section{RESULTATS}

\section{Teneur en Matière Sèche (MS)}

L'analyse de la variance des teneurs en matière sèche de la morelle a révélé des différences significatives $(\mathrm{P}<0,05)$ entre les sites et régions concernées. Les données obtenues indiquent que le taux de MS varie entre 5 et $32,50 \%$ respectivement pour les échantillons de Muea et Ekona dans la région du Sud-ouest $(5,00 ; 5,33 ; 7,33 ; 13,33$; 32,50). Dans le Centre, à l'exception d'Etoug-Ebe dont la valeur est de 15,16\%, tous les autres sites ont des taux de MS supérieurs à $15 \%$. Toutefois, sur le plan de la matière sèche, quelques sites révèlent des similarités (Tableau 2).

\section{Teneur en cendres}

Concernant les teneurs en cendres, des différences significatives $(\mathrm{p}<0,05)$ ont été observées dans les sites et régions. Les valeurs étaient particulièrement élevées dans la région du Centre (Tableau 2). Les taux de cendres variaient de $23,01 \%$ à $32,29 \%$ (Centre) ; $15,50 \%$ à $25,90 \%$ (Sud-Ouest); 19,85 à $22,15 \%$ (Nord) contre 17,04 à 19,03\% (Littoral) et de $17,52 \%$ à 22,85\% (Ouest). 


\section{Teneur en protéines brutes}

L'analyse statistique des protéines brutes a montré une différence significative au seuil de 5\% entre les régions ainsi que les sites dans la même région (Tableau 2). Les valeurs obtenues variaient de 25,63\% à 72,64\% respectivement pour Mokonje (Sud-Ouest) et Koupara 2 (Ouest).

\section{Teneur en phosphore}

Les niveaux de phosphore étaient relativement élevés et variaient entre 6,01 $\mathrm{g} / \mathrm{kg}$ de MS et $92,56 \mathrm{~g} / \mathrm{kg}$ de MS. L'analyse de la variance a montré que les moyennes étaient significativement différentes $(\mathrm{p}<0.05)$ dans les régions et les sites (Tableau 2 ).

\section{Teneur en Calcium}

Les teneurs en calcium des échantillons de morelle africaine étaient significativement différentes entre les sites et variaient de 4,30 à $34,85 \mathrm{~g} / \mathrm{kg}$ de MS respectivement pour les sites Kossala et Koupara1. Les sites de Kossala, Kismatari et Pitoa présentaient des teneurs inférieures à $10 \mathrm{~g} / \mathrm{kg}$. La relation entre $\mathrm{Ca}$ et $\mathrm{P}$ a révélé un rapport $\mathrm{Ca} / \mathrm{P}$ de 0,05 à 4,63 ; ce rapport dans 13 sites restait inférieur à 0,5 (Tableau 2). C'est le cas dans tous les sites des régions du Centre et du Littoral, mais aussi dans 02 sites du Sud-Ouest et 01 site du Nord.

\section{Teneur en Fer}

Les résultats de cette étude ont montré des différences significatives entre les différents sites avec 08 sites ayant le plus haut niveau de teneur en fer (> $50 \mathrm{mg} / 100 \mathrm{~g}$ ) dans les feuilles (Figure 3). Les sites de l'Ouest se sont distingués avec des teneurs en fer plus importantes par rapport aux autres régions. Les valeurs variaient de 67,21 mg/100g à 153 , $61 \mathrm{mg} / 100 \mathrm{~g}$; par contre le Sud-Ouest a présenté des valeurs comprises entre 19,36 $\mathrm{mg} / 100 \mathrm{~g}$ et $33,08 \mathrm{mg} / 100 \mathrm{~g}$.

\section{Teneur en Cuivre}

L'analyse de la variance a révélé des différences significatives entre les régions et les sites $(p<0,05)$. Seuls les sites d'Ekona et Woya-Mavio ainsi que Kossala et Nkolodom n'étaient pas différents entre eux. Les teneurs en cuivre ont varié entre 8,93 et $34,82 \mathrm{mg} / \mathrm{kg}$ respectivement pour Mokonje et Koupara 2. En général, les valeurs dans la majorité des sites ont été supérieures au seuil de toxicité de $15 \mathrm{mg} / \mathrm{kg}$ de MS fixé par l'OMS. Cependant, 10 échantillons se sont retrouvés avec des valeurs inférieures à $15 \mathrm{mg} / \mathrm{kg}$ de MS ; il s'agit de tous les sites de production du SudOuest (Mokonje- 8,93 ; Ekona 9,82 ; WoyaMavio 9,76 ; Kassala- 12,14 ; Muea 11,46) ; de 2 sites du Littoral (Mbouroukou1 9, 42 ; Périurbain Douala IV 9,58) et de 3 sites du Centre (Etoug Ebe 13,68 ; Nkolbisson 13,49 ; Nkolodom 12,17) (Figure 4).

Tableau 1: Rapport Ca/P dans les échantillons de morelle africaine.

\begin{tabular}{lrrrrr}
\hline Echantillons & Centre & Littoral & Sud-Ouest & \multicolumn{1}{c}{ Ouest } & \multicolumn{1}{c}{ Nord } \\
\hline 1 & $\mathbf{0 , 3 2 7}$ & $\mathbf{0 , 3 2 7}$ & 1,440 & 2,196 & 2,315 \\
2 & $\mathbf{0 , 2 7 4}$ & $\mathbf{0 , 2 5 4}$ & $\mathbf{0 , 0 4 6}$ & 2,515 & 2,190 \\
3 & $\mathbf{0 , 2 7 8}$ & $\mathbf{0 , 4 7 6}$ & $\mathbf{0 , 1 5 1}$ & 4,629 & 0,508 \\
4 & $\mathbf{0 , 3 6 2}$ & $\mathbf{0 , 2 4 4}$ & 1,847 & 4,598 & 2,033 \\
5 & $\mathbf{0 , 2 1 9}$ & $\mathbf{0 , 1 5 8}$ & 1,222 & 2,707 & $\mathbf{0 , 4 6 0}$ \\
\hline
\end{tabular}


Tableau 2 : Composition moyenne de la morelle des différents sites.

\begin{tabular}{|c|c|c|c|c|c|c|}
\hline Regions & Sites & Matière sèche (\%) & Cendres (\%) & Protéines (\%) & Phosphore (g/kg) & Calcium (g/kg) \\
\hline \multirow[t]{5}{*}{ Centre } & Etoug E & $15,16 \pm 0,00^{f}$ & $23,01 \pm 0,08^{h}$ & $45,68 \pm 0,01^{\mathrm{r}}$ & $48,66 \pm 0,00^{\mathrm{i}}$ & $15,92 \pm 0,00^{i}$ \\
\hline & Ezazou & $19,53 \pm 0,00^{\mathrm{m}}$ & $26,36 \pm 0,97^{\mathrm{i}}$ & $41,74 \pm 0,00^{1}$ & $67,40 \pm 0,00^{\mathrm{m}}$ & $18,47 \pm 0,00^{\mathrm{k}}$ \\
\hline & Mokolo & $28,26 \pm 0,00^{1}$ & $30,17 \pm 0,03^{\mathrm{j}}$ & $32,31 \pm 0,01^{\mathrm{d}}$ & $73,46 \pm 0,00^{\mathrm{n}}$ & $20,42 \pm 0,00^{1}$ \\
\hline & Nkolbisson & $21,25 \pm 0,00^{\mathrm{k}}$ & $32,29 \pm 1,24^{\mathrm{k}}$ & $38,34 \pm 0,03^{h}$ & $58,35 \pm 0,00^{\mathrm{k}}$ & $21,13 \pm 0,00^{1}$ \\
\hline & Nkolodom & $24,28 \pm 0,00^{\mathrm{j}}$ & $24,03 \pm 0,05^{1}$ & $42,78 \pm 0,02^{\circ}$ & $64,09 \pm 0,00^{1}$ & $14,04 \pm 0,00^{\mathrm{f}}$ \\
\hline \multirow[t]{5}{*}{ Littoral } & Loum & $23,75 \pm 0,26^{\mathrm{j}}$ & $17,34 \pm 0,37^{\mathrm{ab}}$ & $47,23 \pm 0,86^{\mathrm{t}}$ & $48,36 \pm 0,00^{\mathrm{i}}$ & $15,82 \pm 0,00^{\mathrm{i}}$ \\
\hline & Mbroukou1 & $18,42 \pm 0,00^{\mathrm{i}}$ & $18,41 \pm 0,24^{\mathrm{d}}$ & $50,59 \pm 0,03^{w}$ & $55,96 \pm 0,00^{\mathrm{j}}$ & $14,23 \pm 0,00^{\mathrm{f}}$ \\
\hline & Mbroukou2 & $12,45 \pm 0,10^{\mathrm{cd}}$ & $19,03 \pm 0,04^{\mathrm{e}}$ & $49,2 \pm 0,01^{\mathrm{v}}$ & $35,89 \pm 0,01^{\mathrm{h}}$ & $17,09 \pm 0,00^{\mathrm{j}}$ \\
\hline & Njombé & $18,08 \pm 0,52^{\mathrm{hi}}$ & $17,04 \pm 0,00^{\mathrm{a}}$ & $48,33 \pm 0,02^{\mathrm{u}}$ & $55,65 \pm 0,00^{\mathrm{j}}$ & $13,58 \pm 0,00^{\mathrm{f}}$ \\
\hline & PériurbDla & $13,00 \pm 0,35^{\mathrm{de}}$ & $18,98 \pm 0,02^{\mathrm{e}}$ & $40,54 \pm 0,01^{\mathrm{j}}$ & $64,79 \pm 0,00^{1}$ & $10,25 \pm 0,01^{\mathrm{d}}$ \\
\hline Sud- & Ekona & $32,50 \pm 1,20^{\mathrm{n}}$ & $25,90 \pm 0,06^{\mathrm{i}}$ & $34 \pm 0,01^{\mathrm{e}}$ & $10,21 \pm 0,00^{\mathrm{e}}$ & $14,66 \pm 0,00^{\mathrm{g}}$ \\
\hline \multirow[t]{4}{*}{ Ouest } & Kossala & $13,33 \pm 0,01^{\mathrm{e}}$ & $15,50 \pm 0,06^{\mathrm{m}}$ & $27,26 \pm 0,01^{\mathrm{b}}$ & $92,56 \pm 0,00^{\mathrm{p}}$ & $4,29 \pm 0,00^{\mathrm{a}}$ \\
\hline & Mokonje & $7,33 \pm 0,12^{\mathrm{b}}$ & $17,75 \pm 0,08^{\mathrm{bc}}$ & $25,63 \pm 0,00^{\mathrm{a}}$ & $86,19 \pm 0,00^{\circ}$ & $12,98 \pm 0,00^{\mathrm{e}}$ \\
\hline & Muea & $5,00 \pm 0,00^{\mathrm{a}}$ & $22,85 \pm 0,11^{\mathrm{h}}$ & $36,61 \pm 0,00^{\mathrm{g}}$ & $8,98 \pm 0,00^{\mathrm{d}}$ & $16,59 \pm 0,00^{\mathrm{j}}$ \\
\hline & Woya-Mavio & $5,33 \pm 0,04^{\mathrm{a}}$ & $19,45 \pm 0,15^{\mathrm{ef}}$ & $28,44 \pm 0,05^{\mathrm{c}}$ & $11,11 \pm 0,00^{\mathrm{f}}$ & $13,58 \pm 0,00^{\mathrm{f}}$ \\
\hline \multirow[t]{5}{*}{ Ouest } & Baigon1 & $9,14 \pm 0,56^{\mathrm{q}}$ & $18,25 \pm 0,09^{\mathrm{cd}}$ & $44,79 \pm 0,01^{\mathrm{q}}$ & $6,01 \pm 0,00^{\mathrm{a}}$ & $13,20 \pm 0,00^{\mathrm{e}}$ \\
\hline & Baigon2 & $11,96 \pm 1,66^{\mathrm{c}}$ & $19,46 \pm 0,17^{\mathrm{ef}}$ & $60,04 \pm 0,03^{x}$ & $6,21 \pm 0,00^{\mathrm{a}}$ & $15,62 \pm 0,00^{h}$ \\
\hline & Foumbot & $15,88 \pm 0,30^{\mathrm{g}}$ & $22,85 \pm 0,02^{\mathrm{h}}$ & $45,89 \pm 0,00^{\mathrm{s}}$ & $7,33 \pm 0,00^{b}$ & $33,93 \pm 0,00^{\mathrm{n}}$ \\
\hline & Koupara1 & $15,45 \pm 0,79^{\mathrm{fg}}$ & $19,45 \pm 0,00^{\mathrm{ef}}$ & $41,97 \pm 0,00^{\mathrm{m}}$ & $7,58 \pm 0,01^{\mathrm{bc}}$ & $34,85 \pm 0,00^{\mathrm{m}}$ \\
\hline & Koupara2 & $15,60 \pm 0,11^{\mathrm{fg}}$ & $17,52 \pm 0,01^{\mathrm{ab}}$ & $72,64 \pm 0,01^{\mathrm{y}}$ & $7,11 \pm 0,00^{b}$ & $19,25 \pm 0,00^{\mathrm{kl}}$ \\
\hline \multirow[t]{5}{*}{ Nord } & Bocklé Lélé & $17,66 \pm 0,01^{\mathrm{h}}$ & $20,12 \pm 0,00^{\mathrm{g}}$ & $39,98 \pm 0,01^{\mathrm{i}}$ & $8,02 \pm 0,00^{c}$ & $18,57 \pm 0,01^{\mathrm{k}}$ \\
\hline & Bocklé Massa & $13,12 \pm 0,01^{\mathrm{e}}$ & $19,85 \pm 0,02^{\mathrm{fg}}$ & $34,76 \pm 0,00^{\mathrm{f}}$ & $6,67 \pm 0,00^{\mathrm{ab}}$ & $14,61 \pm 0,00^{\mathrm{g}}$ \\
\hline & Kismatari & $7,66 \pm 0,22^{b}$ & $22,15 \pm 0,00^{\mathrm{n}}$ & $41,31 \pm 0,00^{\mathrm{k}}$ & $16,58 \pm 0,00^{\mathrm{g}}$ & $8,42 \pm 0,00^{\mathrm{c}}$ \\
\hline & Ourolabo & $10,17 \pm 0,02^{\circ}$ & $21,15 \pm 0,00^{\circ}$ & $42,23 \pm 0,01^{\mathrm{n}}$ & $9,15 \pm 0,00 \mathrm{~d}^{\mathrm{e}}$ & $18,60 \pm 0,00^{\mathrm{k}}$ \\
\hline & Pitoa & $10,97 \pm 0,02^{p}$ & $20,15 \pm 0,02^{\mathrm{g}}$ & $43,06 \pm 0,01^{\mathrm{p}}$ & $11,21 \pm 0,00^{\mathrm{f}}$ & $5,16 \pm 0,00^{\mathrm{b}}$ \\
\hline
\end{tabular}

Les chiffres en colonne ayant les mêmes lettres ne sont pas significativement différents au seuil de 5\%. 


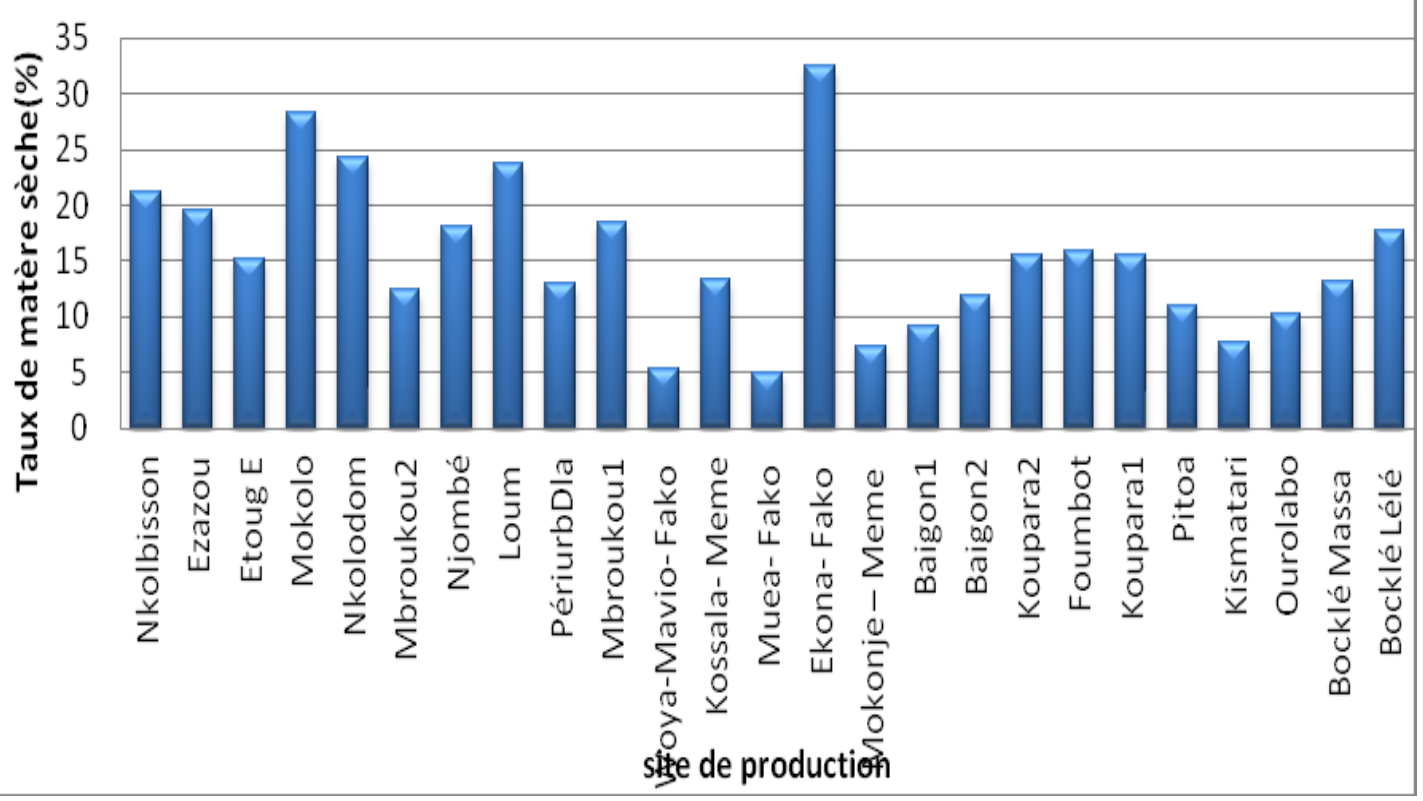

Figure 1: La teneur en matière sèche de la morelle dans les différents sites de production.

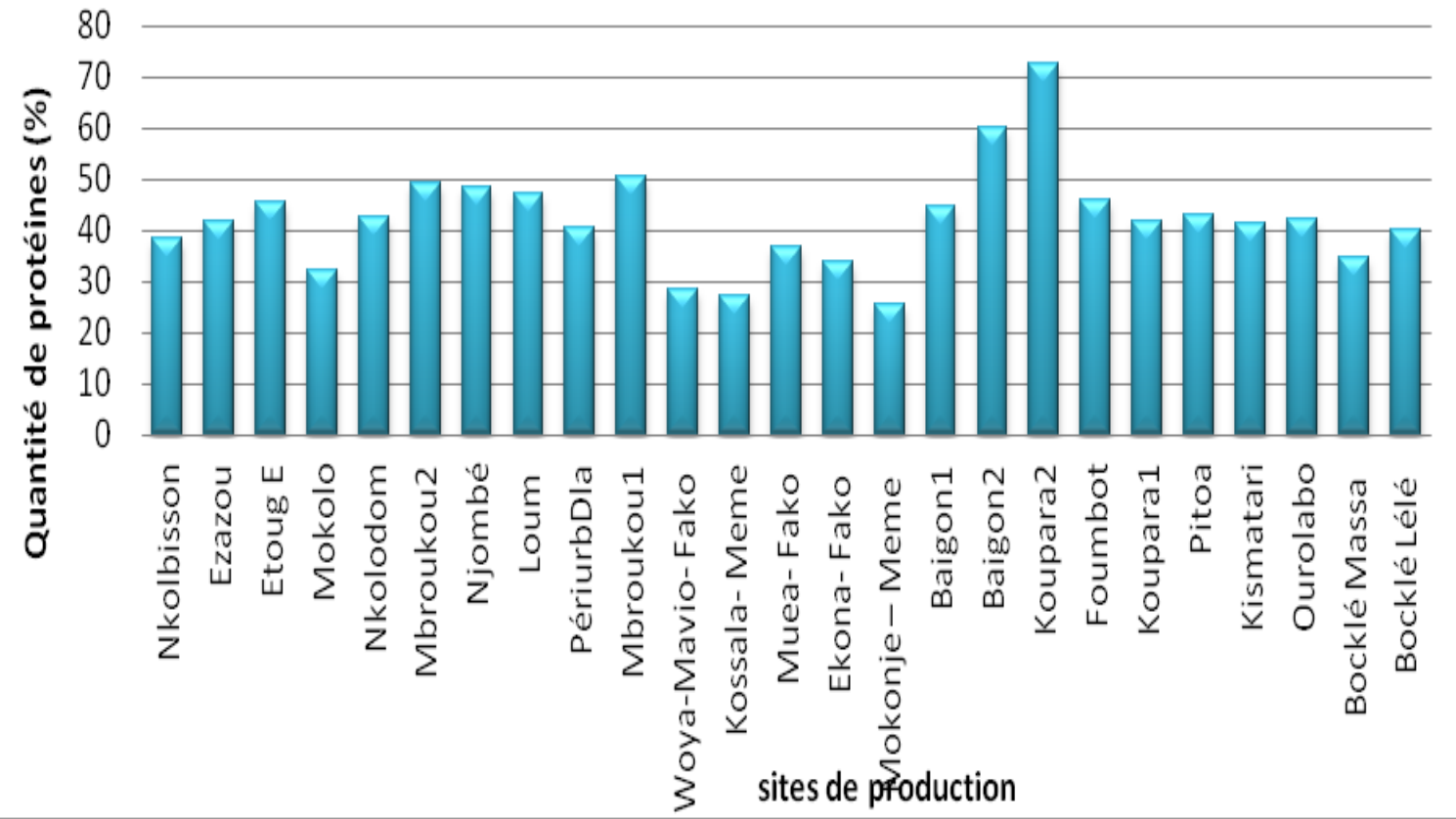

Figure 2 : Teneur en protéines des échantillons. 


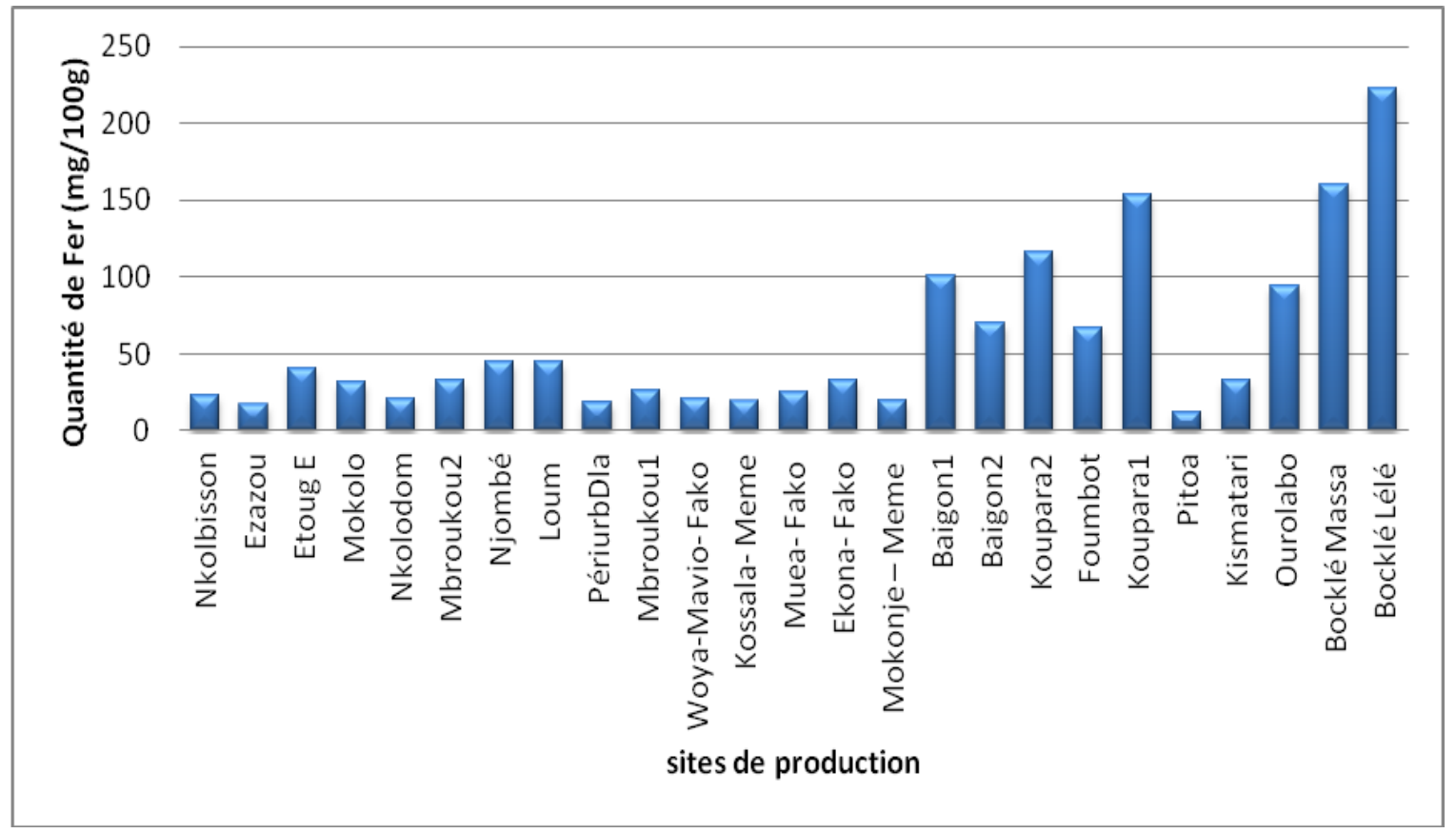

Figure 3 : Teneurs en fer dans les échantillons de morelle africaine.

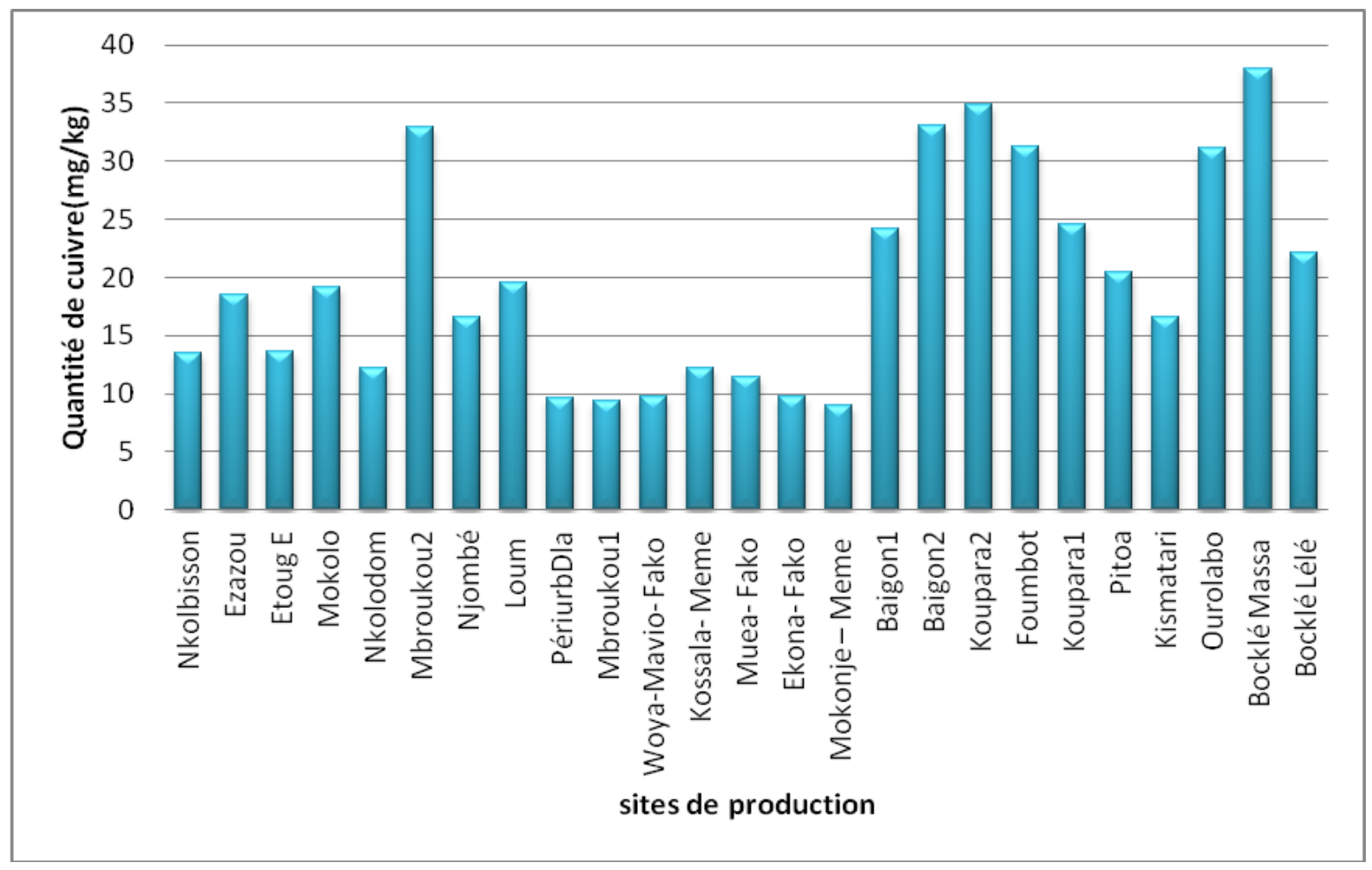

Figure 4 : Teneurs en cuivre dans les échantillons de morelle africaine. 


\section{DISCUSSION}

Les résultats de cette étude confirment que la teneur en eau de la morelle est élevée, mais variable d'une région à une autre avec pour corollaire une MS relativement faible dans les légumes des différents sites. Ces résultats sont semblables à ceux obtenus par divers auteurs comme Kwenin et al. (2011) pour les feuilles de Talinum triangulare "Bokoboko" (8,17\%), Moringa oleifera (25\%) consommées au Ghana et Vodouhe et al. (2012) pour les feuilles de Solanum macrocarpum $(11,01 \%)$ et Amaranthus hybridus (19,53\%) acclimatées au Bénin. En effet, la MS des légumes du Sud-Ouest est moins importante que celle des légumes des autres régions notamment ceux du Centre. Ceci pourrait se justifier par l'itinéraire technique de production, la fertilisation ou les dates de récolte des légumes. SossaVihotogbe et al. (2013) ont rapporté un effet significatif de la fertilisation sur la teneur en eau de $S$. radiatum, $C$. sesamoïdes and J.tenella, ces données corroborent les résultats obtenus dans le cadre de cette étude. Ayant travaillé avec la morelle en préfloraison, certains échantillons, notamment ceux du Sud-Ouest, auraient été plus jeunes que d'autres dans la croissance ou pourraient rappeler l'effet de la fertilisation sur la culture de la morelle africaine. En effet, la récolte intervenait à partir de 45 jours après semis ; Diouf M. et al. (2017) ont présenté dans leurs différentes études les périodes idéales de récolte pour la disponibilité des éléments nutritifs. Car selon Fondio et al. (2013), l'accumulation de la matière sèche et la teneur en éléments nutritifs sont progressives et évoluent selon les saisons, la fertilisation et les stades de développement des plantes. Les résultats de cette étude pourraient servir d'indicateurs des pratiques culturales dans les différents sites de production. Les taux de MS de la morelle de la plupart des sites corroborent les résultats de l'investigation de la FAO (2006) qui montrent que les teneurs en MS des légumes feuilles varient entre $10 \%$ et $40 \%$.
Les taux de cendres sont plus élevés, que ceux obtenus par Oulai et al. (2014) pour Amaranthus hybridus (8,59\%) et Ceiba pentandra $(25,67 \%)$ consommés dans le Nord de la Côte d'Ivoire. Les teneurs en cendres considérées comme moins importantes dans certains sites se révèlent plus grandes que les valeurs élevées obtenues par Vodouhe et al. (2012) pour A. hybridus (18,93\% MS). A ce titre, la morelle africaine des sites d'étude peut être considérée comme une bonne source de minéraux en comparaison avec les valeurs (2 - 10\%) obtenues pour les céréales et les tubercules (FAO, 2006).

Les teneurs en protéines de la morelle des différents sites sont plus élevées que celles rapportées pour la plupart des légumes feuilles tels que Momordica balsamina (11.29\%) et Moringa oleifera $(20.72 \%)$ considérés comme importantes sources de protéines (Asaolu et al., 2012). En dehors du Sud-Ouest $(30,39 \%)$ qui possède des teneurs en protéines inférieures à celles $(37,5 \%)$ observées dans les légumes-feuilles consommés en côte d'Ivoire et au Nigeria (Onwordi et al., 2009; Ochoanin et al., 2012), la majorité des sites présente des légumes plus riches en protéines $(42,22 \%)$ que ceux $(41,66 \%)$ analysés par Ponka et al. (2005) ou ceux étudiés au Bangladesh par Miah et al. (2015). Ces valeurs indiquent que les échantillons de morelle africaine analysés peuvent être une bonne source de protéines. Selon Sheetal et al. (2005), à plus de $12 \%$ de protéines dans les végétaux, ces derniers constituent une bonne source de protéines et peuvent contribuer à assurer l'équilibre alimentaire des populations pauvres. Les taux importants observés dans la région de l'Ouest $(41,97 \%$; 44,79\%; $45,89 \% ; 60,04 \% ; 72,64 \%)$ pourraient être attribués à la fertilisation azotée qui en accord avec les travaux de Agbo et al. (2009) ; SossaVihotogbe et al. (2013) influence la teneur en protéines.

Les valeurs de calcium obtenues dans cette étude 430 à $3485 \mathrm{mg} / 100 \mathrm{~g}$ (Tableau 2) sont supérieures aux teneurs en calcium $(9,00$ - 181) $\mathrm{mg} / 100 \mathrm{~g}$ obtenues par Miah et al. (2015) pour les légumes consommés au 
Pakistan. Néanmoins, ces valeurs sont inférieures à celles $(1211-4680 \mathrm{mg} / 100 \mathrm{~g}) \mathrm{de}$ Oulai et al. (2014) pour les légumes-feuilles consommés dans le Nord de la Côte d'Ivoire, et 23 sites de production présentent une teneur en calcium inférieure à $22,60 \mathrm{~g} / \mathrm{kg}$ de $\mathrm{MS}$ obtenue par Ocho-anin et al. (2012). Ces teneurs en calcium relativement élevées dans les échantillons seraient importantes pour les populations car le calcium est un facteur majeur dans la fortification des os. De plus, il joue un rôle dans la coagulation du sang et l'absorption de la vitamine B12 (Mensah et al., 2008).

Les teneurs en phosphore de cette étude diffèrent de celles obtenues par Grubben et al. (2004) et Ocho-anin et al. (2012), qui présentent respectivement des valeurs relativement basses $(75 \mathrm{mg} / 100 \mathrm{~g} \mathrm{MS})$ et très élevées (2229 mg/100g de MS) dans les feuilles de morelle africaine. Ces différences pourraient être liées aux matières fertilisantes qui possèdent le phosphore (NPK) et sont utilisées par les producteurs d'une part ainsi qu'à la fertilité des sols des différents sites de production d'autre part.

$\mathrm{La}$ relation entre $\mathrm{Ca}$ et $\mathrm{P}$ révèle un rapport $\mathrm{Ca} / \mathrm{P}$ de 0,05 à 4,63 ; ce rapport dans 13 sites de production reste inférieur à 0,5 (Tableau 1). C'est le cas dans tous les sites de production du Centre et du Littoral, mais aussi dans 02 sites du Sud-Ouest et 01 site du Nord. Selon Adeyeye et al. (2005), un rapport $\mathrm{Ca} / \mathrm{P}$ supérieur à 1 dans un aliment serait avantageux sur le plan nutritionnel car ces aliments seraient considérés comme riches en Ca. Ainsi la morelle de la région de l'Ouest $(\mathrm{Ca} / \mathrm{P}>1)$ serait donc meilleure que celle des régions du Centre et du Littoral pour son apport en calcium.

Le niveau de fer dans les échantillons de l'Ouest est au moins le double du niveau des échantillons du Sud-Ouest. Ceci pourrait se justifier par la nature des sols impliqués dans la culture de la morelle dans les différentes régions d'une part, l'itinéraire technique et les pratiques culturales d'autre part. En effet, l'ion ferreux $\mathrm{Fe}^{++}$absorbé par les racines peut être transformé en oxyde ferrique inassimilable dans les sols calcaires et alcalins; la plupart des micronutriments deviennent moins disponibles avec l'augmentation du pH du sol. Ainsi, les sols du Sud-Ouest seraient basiques ou auraient reçu trop de fertilisants dont les éléments pourraient immobiliser le fer (SossaVihotogbe et al., 2013). En général, ces résultats sont plus élevés que ceux obtenus par Vodouhe et al. (2012) pour les légumes acclimatés au Bénin $(2,1 \mathrm{mg} / 100 \mathrm{~g} \quad S$. macrocarpum; $2,3 \mathrm{mg} / 100 \mathrm{~g}$ A. hybridus et 1,5 $\mathrm{mg} / 100 \mathrm{~g}$ O. gratissimum). Ces valeurs sont également supérieures aux teneurs en fer obtenues par Kwenin et al. (2011) pour les légumes traditionnels consommés au Ghana $(14,64 \mathrm{mg} / 100 \mathrm{~g}$ Xanthosoma sagittifolia; 40,50 mg/100 g Amaranth cruentus; 28,21 $\mathrm{mg} / 100 \mathrm{~g}$ Talinum triangulare). La teneur importante en fer dans la morelle africaine de cette étude est indispensable aux consommateurs, car le fer joue un rôle important dans la formation de l'hémoglobine $\mathrm{du}$ sang et dans le déroulement des réactions du métabolisme (Geissler et al., 2005). Ainsi, la morelle africaine des différents sites pourrait contribuer à l'amélioration des besoins en fer dans l'organisme des populations vulnérables.

Le cuivre est un important oligoélément qui prend part aux activités des enzymes dans l'organisme. Le niveau de $\mathrm{Cu}$ dans la morelle africaine des sites de cette étude est plus élevé (Figure 4) que les valeurs rapportées pour certains légumes du Nigeria (Onwordi et al., 2009).

Par ailleurs, les résultats de la région de l'Ouest $(24,20 ; 33,06 ; 31,21 ; 24,60$; $34,82)$ et ceux du Nord $(22,06 ; 38,01 ; 16,60$; $31,07 ; 20,40)$ ont largement dépassé 15 $\mathrm{mg} / \mathrm{kg}$ de MS seuil de toxicité fixé par l'OMS (Temgoua et al., 2015). En plus, les valeurs sont supérieures aux résultats de Lubumbashi pour l'amarante $(21,90 \mathrm{mg} / \mathrm{kg})$ et la poirée bette $(24,32 \mathrm{mg} / \mathrm{kg})$ qui ont été produits dans des sols contaminés en élément trace métallique (ETM) (Mpundu et al., 2013). L'utilisation d'importantes quantités de pesticides par les producteurs de légumes 
pourrait expliquer les valeurs observées. En effet, les informations reçues des producteurs de morelle africaine ont ressorti que les producteurs $(88,10 \%)$ traitent les légumes feuilles à l'aide de fongicides et d'insecticides, tandis que $11,35 \%$ n'appliquent pas de pesticide (Ngo Bogmis, 2015). Par ailleurs, de nombreux maraîchers ont attesté ne pas connaître les risques liés aux pratiques locales utilisées et ignorer les risques de contamination des légumes par les exploitants maraîchers. Ces résultats sont en accord avec ceux rapportés par Wognin et al. (2013) pour le cas d'Abidjan sur le niveau de connaissances des producteurs des risques sanitaires du maraîchage. Les principaux produits phytosanitaires utilisés dans les sites de production maraîchère sont les organophosphorés (Chlorpyriphos Ethyl, Ethoprophos, ...), les carbamates (Nméthylcarbamate de méta-tolyle, ...) et les organochlorés (Oxyde de cuivre et Métalaxyl, Métalaxyl et Mancozèbe, ...). Il s'agit aussi bien des pesticides chimiques recommandés ou non, dont l'utilisation peut provoquer des dangers au niveau de l'environnement et de l'organisme humain du fait de la présence des résidus dans les légumes (Ahouangninou et al., 2012). Toutefois, les travaux de Temgoua et al. (2015) ont montré la pollution des récoltes sans application d'intrant chimique sur la parcelle de cultures maraîchères. Ainsi, le sol du site de production peut être la source de contamination car d'après les travaux de Mpundu et al. (2013), les métaux lourds accumulés sont absorbés par les légumes pendant leur développement. Par conséquent, les teneurs en $\mathrm{Cu}$ dans ces échantillons de morelle africaine devraient conduire à une évaluation des sols dans les différents sites de collecte afin de déterminer leur niveau de contamination et d'imprégnation en ETM.

\section{Conclusion}

L'étude a permis de connaître les caractéristiques de la morelle africaine des différents sites de production ainsi que la qualité nutritive des légumes dans les régions concernées. Les teneurs en éléments nutritifs sont importantes et variables d'un site à un autre. Les taux de MS et de cendres sont élevés dans la plupart des sites. La teneur en protéines dans la morelle collectée varie de facon significative $(\mathrm{P}<0,05)$ d'un site à un autre. Les valeurs sont parfois supérieures à la moyenne de certains légumes feuilles considérés comme importantes sources de protéines. Cela est bénéfique sur le plan nutritionnel. Cependant, les valeurs obtenues peuvent remettre en cause les engrais et le mode de fertilisation pratiqué par les producteurs. La composition minérale des échantillons est également importante et pourrait contribuer de facon significative à l'amélioration de certaines carences nutritionnelles. Mais le $\mathrm{Cu}$, élément trace métallique (ETM) évalué dans les échantillons apparaît à des valeurs maximales voire supérieures aux normes internationales dans toutes les régions. Cependant, les teneurs en $\mathrm{Cu}$ dans les sites de l'Ouest et du Nord sont les plus incriminées. Etant donné les taux élévés de cuivre retrouvés dans ces échantillons, il est recommandé de mener une étude approfondie dans ces régions pour déterminer les pratiques culturales et les itinéraires techniques mis en œuvre par les producteurs. De même, des analyses de sols permettraient de déterminer la composition et la concentration en éléments toxiques des sites de production, par conséquent d'avoir d'amples informations sur les causes et de mieux apprécier les sources possibles de contamination.

\section{CONFLIT D'INTERETS}

Les auteurs déclarent qu'ils n'ont aucun conflit d'intérêts.

\section{CONTRIBUTIONS DES AUTEURS}

NBMN a contribué à l'élaboration du protocole et la collecte des échantillons. Elle a participé aux analyses des données et la rédaction du manuscrit. FAN a contribué à la collecte des échantillons, la revue de l'article et la traduction du résume en anglais. MGA a participé à la rédaction et à l'édition du 
manuscrit. Tous les auteurs ont lu et approuvé le manuscrit.

\section{REMERCIEMENTS}

Les auteurs remercient l'Institut de Recherche Agricole pour le Développement (IRAD) pour sa contribution à la réussite de cette étude.

\section{REFERENCES}

Adeyeye EI, Aye PA. 2005. Chemical composition and the effect of salts on the food properties of Triticum durum whole meal flour. Pakistan Journal of Nutrition, 4(3): 187-196.

Adjatin A. 2006. Contribution à l'étude de la diversité des légumes feuilles traditionnels consommés dans le département de l'Atacora au Togo revue bibliographique Diplôme d'études approfondies. Université de Lomé (Togo), 51p.

Adorgloh R. 2006. Guide pour le développement de l'entreprise de production et de commercialisation de légumes de qualité dans les régions urbaines et périurbaines du Sud Benin Rapport de consultation, IITA Bénin, $86 \mathrm{p}$.

Agbo E, Kouamé C, Mahyao A, N'Zi JC, Fondio L. 2009. Nutritional importance of Indigenous Leafy Vegetables in Côte d'Ivoire. Acta Hort., 806(1): 361- 366. DOI : 10.17660/ActaHortic.2009.806.45

Ahouangninou C, Martin T, Edorh P, BioBangana S, Samuel O, St-Laurent L, Dion S, Fayomi B. 2012. Characterization of Health and Environmental Risks of Pesticide Use in Market-Gardening in the Rural City of Tori-Bossito in Benin, West Africa. Journal of Environmental Protection, 3(3): 241-248 DOI:10.4236/jep.2012. 33030

AOAC. 1990. Association of Official Analytical Chemists. Official Method of
Analysis (15 ${ }^{\text {th }}$ edn), Helrich K (ed). Arlington: Virginia 22201, USA.

Asaolu SS, Adefemi OS, Oyakilome IG, Ajibulu KE, Asaolu MF. 2012. Proximate and mineral composition of Nigerian leafy vegetables. Journal of Food Research, 1(3): 214-218, DOI: http://dx.doi.org/10.5539/jfr.v1n3p214

Batchep R. 2009. Analyse des filières légumes feuilles (amarante, morelle, corète potagère) dans la ville de Yaoundé. Mémoire de fin d'études. FASA. Université de Dschang, 109 p.

Diouf M, Gueye M, Samb PI. 2017. Utilisation des paramètres biochimiques pour la détermination de la date optimale de récolte des feuilles de bissap (Hibiscus sabdariffa L.) au Sénégal. Int. J. Biol. Chem. Sci., 11(5): 2306-2314. DOI :

https://dx.doi.org/10.4314/ijbcs.v11i5.28

FAO. (Food and Agricultural Organization of United Nation) 2006. Proximate composition of foods. Http:/www.fao.org/ag.

Fondio L, Agbo Adouko E, Mahyao A, N'zi J. C, N'gbesso Mako FDP, Djidji AH, Kouame C. 2013. Quelles contributions des légumes feuilles traditionnels à la sécurité alimentaire et à l'allègement de la pauvreté des populations urbaines en Côte d'Ivoire ? Conférence AGRAR 2013 « L'agriculture face aux défis de l'alimentation et de la nutrition en Afrique : Quels apports de la recherche en zones cotonnière » Côte d'Ivoire, Yamoussoukro, 3-7 juin 2013 Projet de Communication 13p.

Geissler CA, Powers HJ. 2005. Human Nutrition $\left(11^{\text {th }}\right.$ edn). Elsevier Churchill: Livingstone.

Grubben GJH, Denton OA. 2004. Plant Resources of Tropical Africa 2. PROTA Foundation : Wageningen, Netherlands. 
Hessou RA. 2006. Guide pour le développement de l'entreprise de production et de commercialisation de légumes de qualité dans les régions urbaines et périurbaines du Sud-Bénin. Rapport de consultation, IITA - Bénin, $86 \mathrm{p}$.

Iheanacho Kizito ME, Udebuani AC. 2009. Nutritional Composition of Some Leafy Vegetables Consumed in Imo State, Nigeria. J. Appl. Sci. Environ. Manage., 13(3): $35-38 . \quad$ DOI : http://www.bioline.org.br/ja

Kwenin WKJ, Wolli M, Dzomeku BM. 2011. Assessing the nutritional value of some African indigenous green Leafy Vegetables in Ghana. Journal of Animal \& Plant Sciences, 10(2): 1300-1305. DOI: http://www.biosciences.elewa.org/ JAPS.

Mensah JK, Okoli RI, Ohaju-Obodo JO, Eifediyi K. 2008. Phytochemical, nutritional and medical properties of some leafy vegetables consumed by Edo people of Nigeria. Afric. J. Biot., 7(14): 2304-2309.

Miah MAS, Mohammed MRLK, Syeda AJ, Nusrat A, Mohammed FI, Badhan S. 2015. Nutritional quality and safety aspects of wild vegetables consume in Bangladesh. Asian Pacific Journal of Tropical Biomedicine, 6(2): 125-131. DOI: http://dx.doi.org/10.1016/j.apjtb. 2015.11.004

Mpundu Mubemba Mulambi M, Useni Sikuzani Y, Ntumba Ndaye F, Muyambo Musaya E, Kapalanga Kamina P, Mwansa M, Ilunga K. 2013. Évaluation des éléments traces métalliques dans les légumes feuilles vendus dans les marchés de la zone minière de Lubumbashi. Journal of Applied Biosciences, 66: 5106- 5113.

Ngo Bogmis M. 2016. Les pratiques de récolte et post récolte de la morelle africaine dans quelques localités du Cameroun. Rapport d'enquête IRAD /C2D, 21p.

Ngom E, Ndjogui TE, Nkongho RN, Iyabano AH, Levang P, Miaro III L, Feintrenie L. 2014. Diagnostic du secteur élæicole au Cameroun. Feintrenie L et Levang P éditeurs. Rapport de synthèse. CIRAD, IRD, CIFOR, WWF-CARPO, Minader.

Ocho-anin Atchibri AL, Soro LC, Kouame C, Agbo EA, Kouadio KKA. 2012. Valeur nutritionnelle des légumes feuilles consommés en Côte d'Ivoire. Int. J. Biol. Chem. Sci., 6(1): 128-135. DOI: http://dx.doi.org/10.4314/ijbcs.v6i1.12

Onwordi CT, Ogungbade AM, Wusu AD. 2009. The proximate and mineral composition of three leafy vegetables commonly consumed in Lagos, Nigeria. Afr J Pure Appl Chem., 3(6): 102-107. http://www.academicjournals.org/AJPA C ISSN 1996 - 0840.

Oulai P, Lessoy Zoue RM, Megnanou R, Doue S. 2014. Proximate composition and nutritive value of leafy vegetables consumed in northern Côte d'Ivoire. European Scientific Journal, 10(6): 212227.

Ponka R, Fokou E, Leke R, Fotso M, Souopgui J, Achu Bih M, Mbiapo Tchouanguep F. 2005. Méthodes de préparation et l'évaluation des plats consommés dans une zone d'endémie palustre au Cameroun (Ngali II). Afr. J. Biotechnol., 4(2): 273-278. DOI: https://doi.org/10.5897/AJB2005.000.30 54

Sheetal G, Jyothi Lakshmi A, Manjunath MN, Jamuna P. 2005. Analysis of nutrient and antinutrient content of underutilized green leafy vegetables. $L W T, \mathbf{3 8}(4)$ : $339-$ 345. DOI: http://dx.doi.org/ 10.1016/j. lwt.2004.06.012.

Sossa-Vihotogbe ANC, Anihouvi BV, Akissoe HN, Amadji LG, Hounhouigan DJ. 2013. Effect of organic and mineral 
fertilization on proximate composition of three leafy vegetables harvested at different periods. Int. J. Biol. Chem. Sci. 7(1): 271-286, DOI: http://dx.doi.org/ 10.4314/ijbcs.v7i1i.23.

Tchiegang C, Kitikil A. 2004. Données ethno nutritionnelles et caractéristiques physico-chimiques des légumes feuilles consommés dans la savane de l'Adamaoua (Cameroun). Tropicultura, 22(1): 11-18.

Tchientche KRKC, Atangana AR, Chagomoka TNR. 2013. Nutritional evaluation of five African indigenous vegetables. Journal of Horticultural Research, 2013.

Temgoua E, Ntangmo TH, Hans-Rudolf P, NJINE T. 2015. Teneur en éléments majeurs et oligoéléments dans un sol et quelques cultures maraichères de la ville de Dschang Cameroun. African crop science journal, 23(1): 35-44.

Vodouhe S, Dovoedo A, Anihouvi VB, Tossou RC, Soumanou MM. 2012. Influence du mode de cuisson sur la valeur nutritionnelle de Solanum macrocarpum, Amaranthus hybridus et Ocimum gratissimum, trois légumes feuilles traditionnels acclimatés au Bénin. Int. J. Biol. Chem. Sci., 6(5): 1926-1937, DOI: http://dx.doi.org/10. 4314/ijbcs.v6i5.3.

Wognin AS, Ouffoue SK, Assemand EF, Tano K, Koffi-Nevry R. 2013. Perception des risques sanitaires dans le maraîchage à Abidjan, Côte d'Ivoire Int. J. Biol. Chem. Sci., 7(5): 1829-1837, DOI: http://dx.doi.org/10.4314/ijbcs. v7i5.4. 